\title{
Trends in cervical cancer mortality
}

Mortality attributable to cervical cancer has been decreasing in the past decades in most developed countries. The steady decline has been largely attributed to early diagnosis and to treatment efficacy when the diagnosis is made in early stages.

In the late 1970s, a few countries reported changes in the trend with moderate increases in the mortality rates among younger women. These observations created some public and medical concern, particularly because they occurred in countries with fairly developed screening programmes. Subsequent analyses identified as possible causes specific failures of the screening programme, notably insufficiency of the population coverage and in the call-recall procedures in place to follow up on abnormal smears. Reinforcement of the screening logistics soon achieved a second reverse of the trend towards a decline.

The attention generated by these results prompted analyses in many countries to assess the existence or else of a similar trend. However, it soon became (repetitiously) clear that mortality trends in cervical cancer are among the most difficult to evaluate in many countries because of (1) the confounding effects of screening, (2) the limited quality of the certification of the cause of death to uterine malignancies, particularly in past decades, and (3) the small number of cases in the younger age groups.

Screening, in a clinical sense, implies taking of a cytological smear. In several countries, including Spain, cytology undertaken in gynaecological practices has increased in recent decades and has probably contributed to early detection and successful treatment of many cases of cervical cancer. Screening programmes in the epidemiological sense defines a centralised public health activity in which all women in a target age range are invited to participate. The latter has proved to be effective in reducing incidence and mortality of cervical cancer and in addition provides information on the coverage (percentage of women in the age groups complying with the proposed screening scheme) offered by the preventive action. In the absence of epidemiologically sound screening programmes, interpretation of time trends is difficult, particularly if an increase in mortality is claimed to exist as indicated by Llorca et al. ${ }^{1}$

Cervical cancer mortality data from regions in Spain have been analysed before. Sanchez Garrido et al published in 1996 a study in which death certificates including three rubrics (cervix (ICD-O 180), uterus (ICD-O 182), and uterus not otherwise specified -NOSICD-O 179)) were jointly analysed for the interval between 1975 and 1992. The study clearly showed that an apparent increase in mortality in Catalonia (an autonomous region in the north east part of Spain) was almost entirely attributable to a time trend reduction of the number of cases coded in the rubric "uterus NOS". When these cases are adequately classified as either "cervix" or "uterus" (as it happened over the time interval), they lead to an apparent increase in the number of cases (and rates) for the two specific cancer sites. A similar conclusion was reached by other authors on data from the entire country, which reported that the fraction of "uterus NOS" for the Spanish population was as high as $80 \%$ in 1975 and still close to $50 \%$ in 1986 (Lopez-Abente $e t a l^{3}$ ).

Recent work on secular trends in cervical cancer incidence has been published or is in preparation focusing on histology specific trends (Vizcaino et $a l^{4}$ and Vizcaino et al, unpublished data). Trends in incidence have some advantages over mortality in that organ specificity is well described and incidence is independent of the results of treatment (although not of the screening coverage). Vizcaino et al conducted an analysis of the data of 60 population-based cancer registries in 25 countries, which included three registries in Spain (Navarra, Zaragoza, and Tarragona). The data base included incidence over the interval 1973-1991 and information on slightly over 175000 cases of cervical cancer (of these, 133000 were squamous cell carcinomas, close to 20000 were adeno and adeno squamous carcinomas, and 22000 were cervical cancer cases with other or without histology). The study identified an underlying upward trend for the cervical adenocarcinomas (about $10 \%$ of the cases) paired with a steady downward trend of the most common type, the squamous cell carcinomas. The authors suggested that adenocarcinomas have an increased chance to escape detection by the routine standard smear because they often arise in the endocervical canal.

The results of these analyses also showed that the incidence of all histological types of cervical cancer remained stable in Spain over the interval of investigation. In the presence of a stable incidence over time, an increase in mortality among the younger cases, as suggested by Llorca et $a l^{1}$ leads to the interpretation that screening practices are poor or that treatment efficacy is decreasing, or both. However, neither of these conclusions is substantiated by the report or the Spanish literature.

Finally, cervical cancer shows a peculiar age specific incidence in which cases below the age of 30 are rare and the occurrence of new cases reaches a plateau after the age 40-45. Because of the small numbers in the age groups below 30, trends that are apparent only in these groups are particularly susceptible to misclassification bias and should be interpreted with greater caution.

Spain has been for a number of years a low risk country for cervical cancer and studies have reported the correspondence with a low average number of sexual partners among women in the precedent decades. Low promiscuity may have contributed in turn, to a low prevalence of human papillomavirus infections and to the low risk of cervical cancer. ${ }^{5}$ The general pattern of sexual behaviour has gradually changed beginning in the 1960s and subsequently. As a consequence, some increases in human papillomavirus related lesions are to be expected after some latency period. However, the crop of human papillomavirus related cervical cancers should mostly consist of pre-invasive lesions and early stage invasive disease. If this is the case, one should expect to first detect such trends in the incidence data and, hopefully, to show limited impact in mortality statistics.

F XAVIER BOSCH

Institut Catalá d'Oncologia, L'Hospitalet, Barcelona, Spain

1 Llorca J, Prieto MD, Delgado-Rodríguez M. Increase in cervical cancer mortality in Spain, 1951-1991. F Epidemiol Community Health 1999;53:408-11.

2 Sanchez MV, Izquierdo A, Beltran M, et al. Tendencias temporales de la mortalidad por cancer de cervix en Cataluña 1975-1992: Analisis del Boletin estadistico de defuncion y del registro de cancer de Girona. Gac Sanit 1996;10:67-72

3 Lopez-Abente G, Pollan M, Ruiz M, et al. Cancer mortality in Spain 1952-1986. Centro Nacional de Epidemiologia. Madrid: Instituto de Salud carlos III, 1992.

4 Vizcaino AP, Moreno V, Bosch FX, et al. International trends in the incidence of cervical cancer I Adenocarcinoma and adenosquamous cell carcinomas. Int f Cancer 1998;75:536-45.

5 Bosch FX, Muñoz N, De Sanjose S, et al. Importance of human papillomavirus endemicity in the incidence of cervical cancer: an extension of the virus endemicity in the incidence of cervical cancer: an extension of the
hypothesis on sexual behavior. Cancer Epidemiol Biomark Prev 1994;3:3759 . 